\title{
Dynamic Colloidal Photonic Crystal Hydrogels with Self-Recovery and Injectability
}

\author{
Yue Ma, ${ }^{1,2,3}$ Peiyan He, ${ }^{2}$ Wanli Xie, ${ }^{1,2}$ Qiang Zhang, ${ }^{4}$ Weiling Yin, ${ }^{1}$ Jianming Pan, \\ Miao Wang, ${ }^{1}$ Xin Zhao $\left({ }^{4},{ }^{4}\right.$ and Guoqing Pan $\mathbb{1}^{1}$ \\ ${ }^{1}$ Institute for Advanced Materials, School of Materials Science and Engineering, Jiangsu University, Zhenjiang, Jiangsu 212013, China \\ ${ }^{2}$ School of Chemistry and Chemical Engineering, Jiangsu University, Zhenjiang, Jiangsu 212013, China \\ ${ }^{3}$ Jiangsu Agrochem Laboratory, Changzhou, Jiangsu 213022, China \\ ${ }^{4}$ Department of Biomedical Engineering, The Hong Kong Polytechnic University, Hung Hom, Hong Kong, China
}

Correspondence should be addressed to Xin Zhao; xin.zhao@polyu.edu.hk and Guoqing Pan; panguoqing@ujs.edu.cn

Received 30 January 2021; Accepted 26 February 2021; Published 30 March 2021

Copyright @ 2021 Yue Ma et al. Exclusive Licensee Science and Technology Review Publishing House. Distributed under a Creative Commons Attribution License (CC BY 4.0).

\begin{abstract}
Simulation of self-recovery and diversity of natural photonic crystal (PC) structures remain great challenges for artificial PC materials. Motivated by the dynamic characteristics of PC nanostructures, here, we present a new strategy for the design of hydrogel-based artificial PC materials with reversible interactions in the periodic nanostructures. The dynamic PC hydrogels, derived from self-assembled microgel colloidal crystals, were tactfully constructed by reversible crosslinking of adjacent microgels in the ordered structure via phenylboronate covalent chemistry. As proof of concept, three types of dynamic colloidal PC hydrogels with different structural colors were prepared. All the hydrogels showed perfect self-healing ability against physical damage. Moreover, dynamic crosslinking within the microgel crystals enabled shear-thinning injection of the PC hydrogels through a syringe (indicating injectability or printability), followed by rapid recovery of the structural colors. In short, in addition to the great significance in biomimicry of self-healing function of natural PC materials, our work provides a facile strategy for the construction of diversified artificial PC materials for different applications such as chem-/biosensing, counterfeit prevention, optical display, and energy conversion.
\end{abstract}

\section{Introduction}

Artificial photonic crystal (PC) materials mimicking the naturally occurring periodic nanostructures in creatures like a chameleon or a butterfly have attracted increasing interest [1-10]. These bioinspired materials have shown great potentials in chem-/biosensing [11-16], bioelectronics [15, 17-19], optical display [20-24], energy conversion [7, 25-27], and others [28-35] due to their unique properties such as brilliant and stable structural colors, slow light, and amplified photon absorption/emission. Although great achievements in artificial PC materials have been made in mimicking the photonic nanoarchitectures in nature, the functional simulation is an area still in its infancy. For instance, the creatures can spontaneously heal from injury and recover their functionality to increase the survivability and lifetime. The simulation of such self-healing property is of critical importance to the PC materials, since accidental cuts and scratches are inevitable during applications, which may deteriorate the performance of these materials. Therefore, artificial PC materials with diversified and complex nanostructures as well as self-recovery capability and mechanical durability are highly sought after.

Amongst various PC materials, PC hydrogels feature the best flexibility and plasticity, considering the fragility of other hard structure-color materials. To import periodic nanostructures in hydrogel networks during fabrication of PC hydrogels, colloidal crystal templating methods were initially employed [36]. After filling, gelling, and etching of the ordered assemblies of hard spheres like silica dioxide, poly(methyl methacrylate), and polystyrene nanoparticles, inverse opal-like PC hydrogels with various structure colors could be obtained [12, 37-39]. Alternatively, PC hydrogels could be prepared by covalent crosslinking of microgel colloidal crystals [40-43]. In contrast to the poor controllability of hard colloidal crystal templating strategy, the postcrosslinking method towards soft microgels enables large-scale and fast 


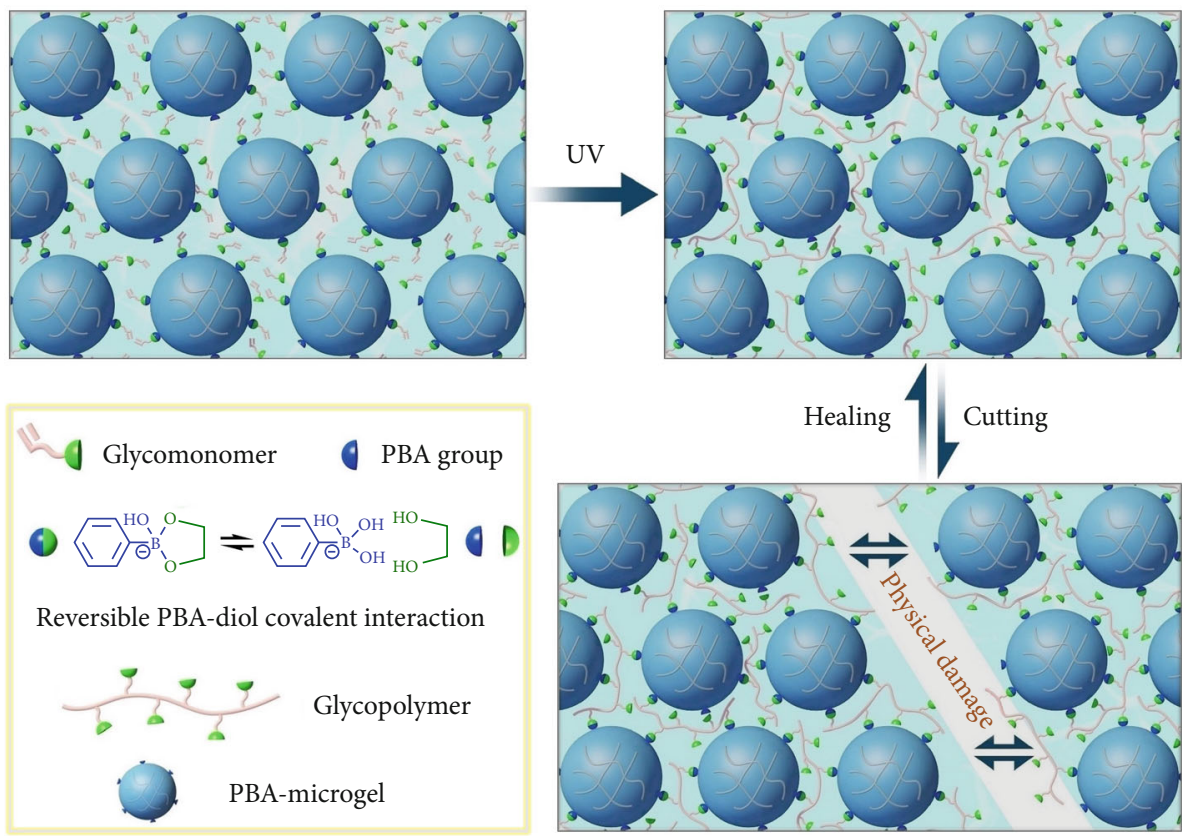

Scheme 1: Schematic illustration of the reversible crosslinking strategy and self-recovery mechanism of the proposed dynamic colloidal PC hydrogels based on dynamic phenylboronate covalent bond.

production of PC hydrogels. Nevertheless, the above two strategies both involve an irreversible solidifying process of the periodic nanostructures that can be permanently damaged by external mechanical stress. In this regard, self-healing hydrogels with nonsupporting inverse opal structures [44] or injectable hydrogels incorporated with photonic supraballs [45] were prepared. These designs indeed endow PC hydrogels with improved survivability or operability. They, however, lack the internally dynamic characteristic of natural PC materials (e.g., self-recovery of biogenic periodic nanostructures on the damaged interfaces after injury) $[4,6]$. Besides, the self-healing mechanisms in these systems mainly relied on the formation of hydrogen bonds, van der Waals forces, or host-guest interactions between the filled hydrogel matrix, whose strength was relatively low for the healing of fracture interface and the maintenance of stable periodic nanostructures [46, 47].

To obtain the internally dynamic PC hydrogels, we shift our focus to the interaction forces of the particle assembly within the microgel colloidal crystals. Although covalent postcrosslinking of adjacent particles can increase their stability to form a colloidal PC hydrogel, the dynamic nature of noncovalently assembled microgel colloidal crystals will be lost. To balance the stability and the dynamics in a colloidal PC hydrogel, here, we propose to use reversible covalent interactions to dynamically stabilize the assembled nanoparticles in microgel colloidal crystals. Such reversible but strong covalent interactions will be achieved by using phenylboronic acid (PBA) derivatives and cis-diols (boron-carbohydrate interaction, Scheme 1) [48-50]. In this context, we intend to prepare microgels with PBA groups and then place them in a glycomonomer solution to self-assemble into ordered colloidal crystals. As PBA is capable of reversible interaction with the cis-diol groups of glycomonomers, photoinitiated poly- merization of the glycomonomer will lead to dynamic crosslinking of adjacent microgel particles though reversible PBAcis-diol interactions between the microgels and the resultant glycopolymers. Such unique dynamic interaction between the particles and the matrix enables the reconfiguration of the assembly units during the self-healing process rather than merely the recombination of the filled hydrogel matrix. In this case, an injectable and self-healing PC hydrogel formed upon dynamic covalently assembled microgel photonic crystals can be obtained. We envision that our dynamic colloidal PC hydrogels will show great potential in constructing diversified and complicated PC materials for novel applications in chem-/biosensing, counterfeit prevention, optical display, and energy conversion.

\section{Results and Discussion}

According to our previous method [51], PBA-containing microgels (PBA-microgels) were synthesized in aqueous solution through surfactant-assistant precipitation polymerization, using sodium dodecyl sulfate (SDS), potassium persulfate (KPS), 4-(2-acrylamidoethylcarbamoyl)-3-fluorophenylboronic acid (AFPBA), N-isopropylacrylamide (NIPAAm), and methylene bisacrylamide (MBAAm) as the surfactant, initiator, PBA functional monomer, backbone monomer, and the crosslinker, respectively (Figure 1(a)). SDS was chosen because it could regulate the sizes of microgels [52], while AFPBA was selected and synthesized as it could reversibly interact with cis-diols (Figure S1) [48]. To achieve dynamic crosslinking in the microgel colloidal crystals, the resultant PBA-microgels were first coincubated with a glucose-derived glycomonomer 3-gluconamidopropyl methacrylamide (GAPMA, Figure S2) and a water-soluble photoinitiator (2-hydroxyethoxy)-2-methylpropiophenone 


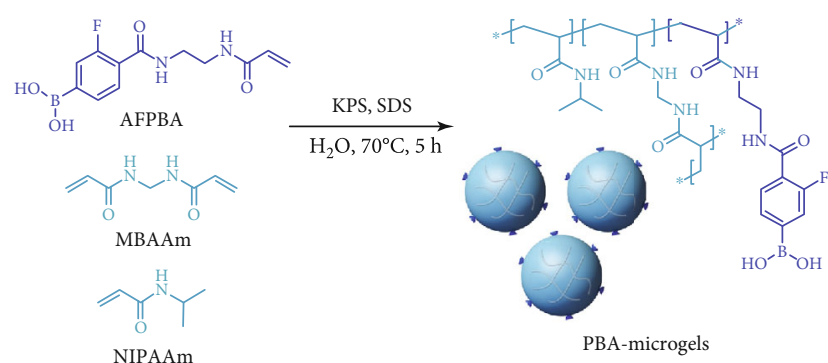

(a)

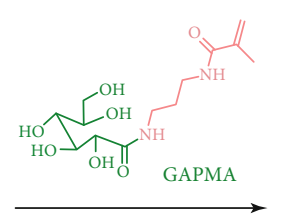

HMP, $15^{\circ} \mathrm{C}$, UV

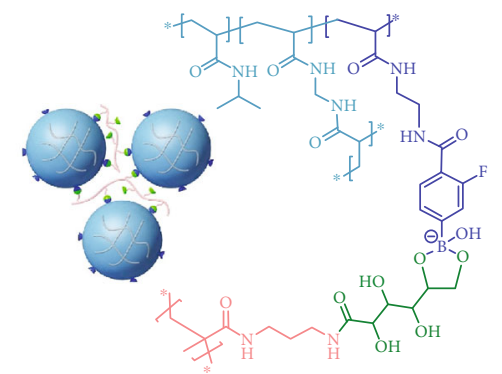

PC hydrogels

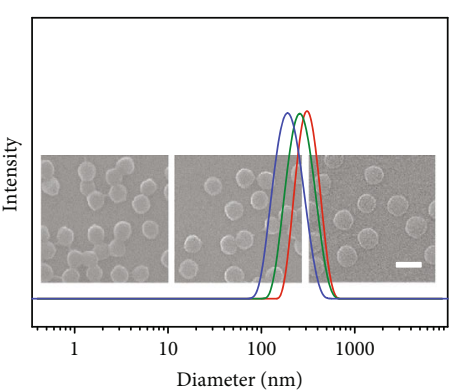

$\begin{aligned}-\mathrm{D}_{\mathrm{H}} & =303 \mathrm{~nm}, \mathrm{PDI}=0.033 \\ \mathrm{D}_{\mathrm{H}} & =250 \mathrm{~nm}, \mathrm{PDI}=0.045\end{aligned}$ $\mathrm{D}_{\mathrm{H}}=185 \mathrm{~nm}, \mathrm{PDI}=0.052$

(c)

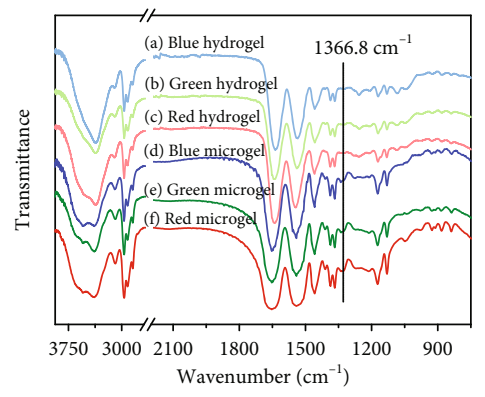

(d)

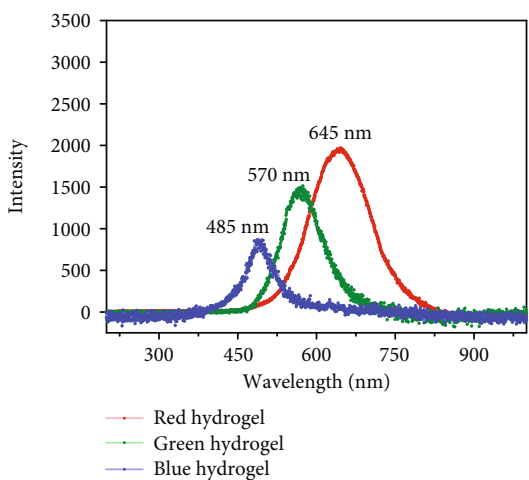

(e)

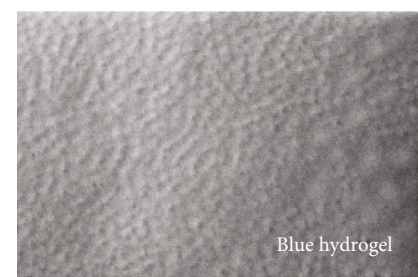

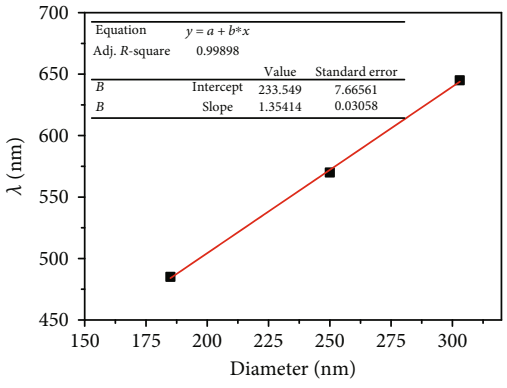

(f)

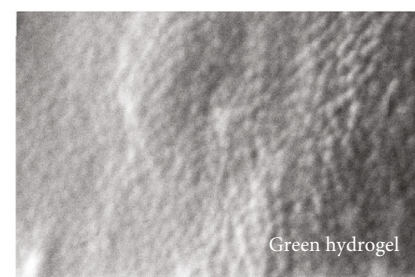

(h)

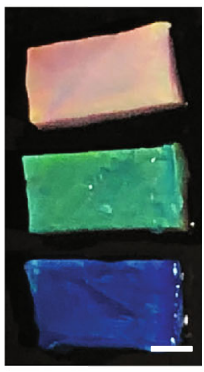

(g)

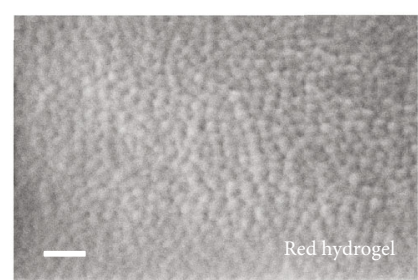

FIGURE 1: (a) Synthesis of the PBA-microgels. (b) Dynamic crosslinking of the preassembled PBA-microgel colloidal crystals via photoinitiated polymerization of cis-diol-containing glycomonomers. (c) Dynamic light scattering (DLS) analysis of the PBA-microgels. Inserts: SEM images showing the morphology of the dry PBA-microgels (average diameters of the PBA-microgels in corresponding SEM images from left to right were 130, 160, and $190 \mathrm{~nm}$ ). Scale bar $=200 \mathrm{~nm}$. (d) FT-IR spectra of the PBA-microgels and PC hydrogels. The reaction between PBA and cis-diol groups in GMAPMA resulted in a weakened peak of B-O stretching $\left(1366.8 \mathrm{~cm}^{-1}\right)$. (e) Reflection spectra and the corresponding photos of three typical colloidal PC hydrogels. (f) The linear relation between the diameter of PBAmicrogels and photonic stopband of the corresponding colloidal PC hydrogels. The reflection peak $(\lambda)$ of PC materials can be estimated by Bragg's equation, $\lambda=2 d_{111} n_{\text {average }}$, in which $d_{111}$ is the interplanar distance of (111) diffracting planes and $n_{\text {average }}$ is the average refractive index of the materials. (g) Photos of the three PC hydrogels. Hydrogel sizes: $1.5 \mathrm{~cm} \times 3.0 \mathrm{~cm}$. Scale bar $=1 \mathrm{~cm}$. (h) SEM images of the lyophilized hydrogels with short-range order in the assembled microgels. Scale bar is $500 \mathrm{~nm}$. 


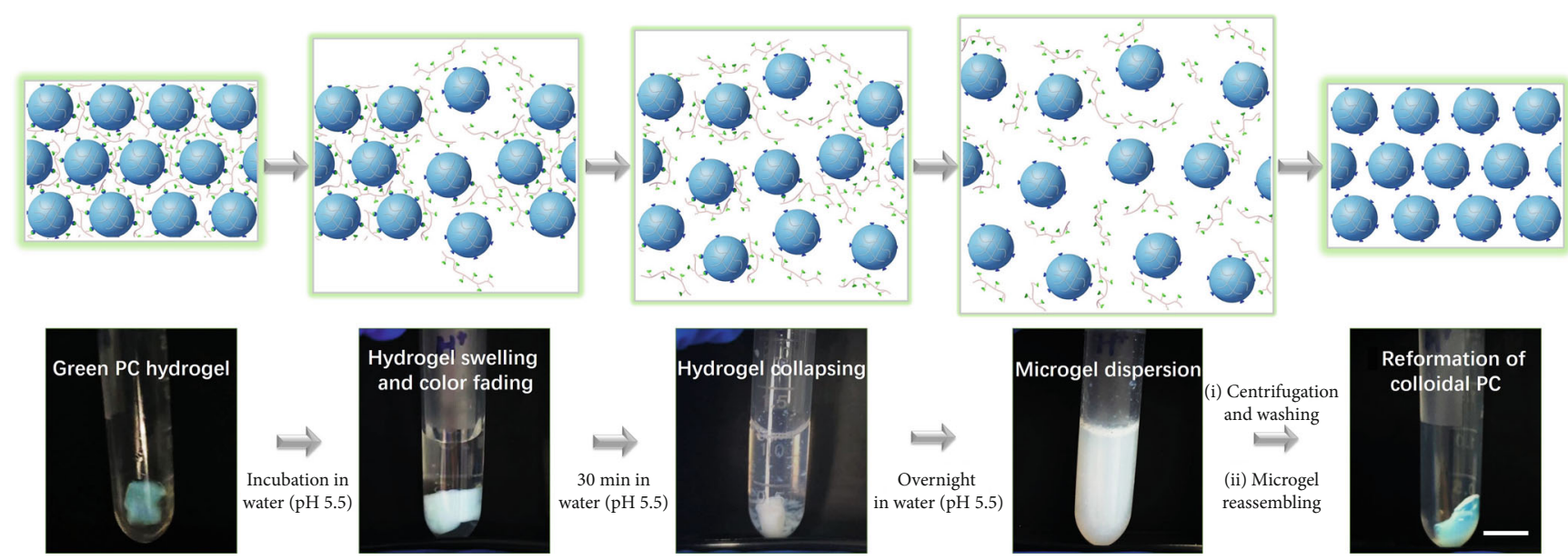

FIGURE 2: Investigation of the internally dynamic characteristics of phenylboronate-crosslinked colloidal PC hydrogel through an acidtriggered disassembly and reassembly method. Scale bar $=1 \mathrm{~cm}$.

(HMP). UV light-initiated polymerization of GAPMA monomers was then switched on when the PBA-microgels self-assembled to ordered structures and showed clear structural colors. Finally, the ordered PBA-microgels formed dynamic colloidal PC hydrogels due to the reversible covalent interactions between the PBA-microgels and the poly(GAPMA) glycopolymers (Figure 1(b)).

To obtain colloidal PC hydrogels with different structural colors, three types of PBA-microgels with different sizes were prepared by regulating the concentrations of SDS and MBAAm (Figure 1(c)). Dynamic light scattering (DLS) analysis indicated that all three microgels showed uniform sizes with diameters increasing from 185, 250 to $303 \mathrm{~nm}$. Scanning electron microscopy (SEM) images revealed that all the microgels exhibited spherical shape. It should be noted that, although the sizes of microgels reflected from DLS and SEM (wet vs. dry) were different, an increasing trend in the diameters could still be observed. Chemical components of the microgels were further characterized using infrared spectroscopy (IR) (Figure 1(d)). In addition to the characteristics of NIPAAm units [53], a characteristic peak of AFPBA (B-O stretching) [54] at $1366.8 \mathrm{~cm}^{-1}$ was also observed in all the spectra. These results indicated the successful preparation of size-different but monodispersed PBA-microgels.

The PBA-microgels were then self-assembled into colloidal photonic crystals in aqueous solution with the glycomonomer GAPMA, following by UV light-initiated polymerization (Figure 1(b)). The weakened peaks of B-O stretching at $1366.8 \mathrm{~cm}^{-1}$ in the IR spectra of three hydrogels confirmed the efficient formation of phenylboronate bonds between PBA and the cis-diols of GAPMA (Figure 1(d)) [55]. As expected, photoinduced crosslinking of the PBAmicrogels with GAPMA efficiently led to the gelation of the systems. As shown in Figures 1(e) and 1(g), three colloidal PC hydrogels with blue $(485 \mathrm{~nm})$, green $(570 \mathrm{~nm})$, and red $(645 \mathrm{~nm})$ structural colors were obtained from the PBAmicrogels with diameters of 185,250 , and $303 \mathrm{~nm}$, respectively. Further study revealed a linear correlation between the microgel sizes and the photonic stop band wavelengths (Figure 1(f)), which was in good agreement with Bragg's law. We then further used SEM to check the assembled microgels in the PC hydrogels (Figure 1(h)). Short-range order could be clearly observed in the arrangement of all three hydrogels, which contributed mainly to the colors reflected on the PC hydrogels. These results together demonstrated that our PBA-based dynamic crosslinking strategy could be used as a general method for the construction of stable colloidal PC hydrogels with diverse structural colors.

Unlike previous PC hydrogels with fixed crystal structures [40-45], reversible phenylboronate-crosslinked strategy would lead to dynamic colloidal PC hydrogels with mobilizable periodic nanostructures. To demonstrate the internally dynamic characteristic of our colloidal PC hydrogel, an acid-induced disassembly and reassembly experiment was carried out (Figure 2). In view of the $\mathrm{pH}$-reversibility of PBA-cis-diol interactions, our phenylboronate-crosslinked colloidal PC hydrogels were found able to disassemble and reassemble by changing the $\mathrm{pH}$ values. As expected, when a piece of green colloidal PC hydrogel was incubated in an acidic aqueous solution ( $\mathrm{pH}$ 5.5), rapid collapsing of the PC hydrogel and subsequently complete dispersion of the microgels into the solution were observed (milky white solution). This is attributed to the reversible crosslinking of the assembled microgels, which enabled acid-induced decrosslinking of the colloidal hydrogels. Moreover, the dispersed microgels could reassemble into colloidal crystals with a green structural color at $\mathrm{pH} 7.5$. These results confirmed that the phenylboronate-crosslinked colloidal PC hydrogels, similar to the natural PC materials, boast the reversibility in the periodic nanostructures, suggesting the potential of self-recovery in practical applications like colorimetric sensors, wearable optical devices, and anticounterfeiting labels.

The dynamic and self-recovery of the three colloidal PC hydrogels were first examined by investigating their selfhealing ability against physical damage (Figure 3 and S3). The blue, green, and red hydrogels were all cut into two halves, and then the two halves were staggered and contacted for self-healing. All the colloidal PC hydrogels showed very fast healing at the interfaces of damage within seconds, and no breakage was found even when the healed hydrogels were 

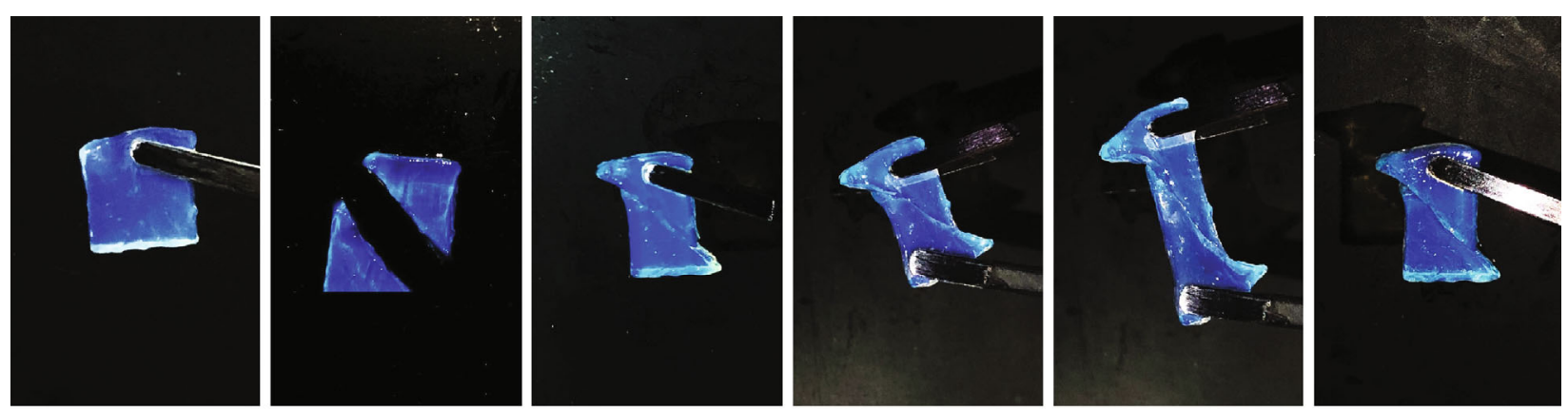

(a)
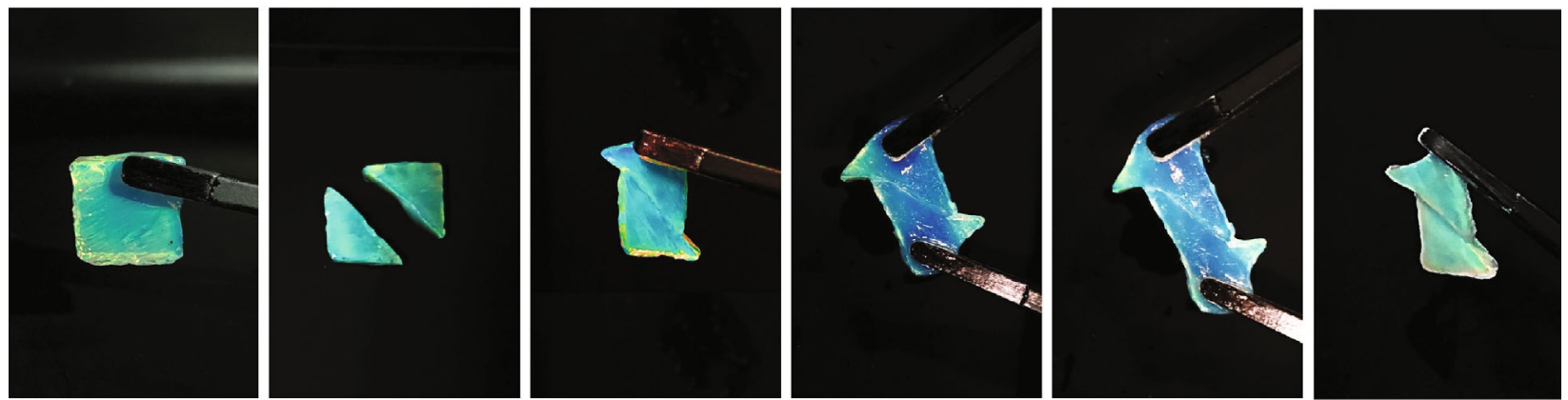

(b)
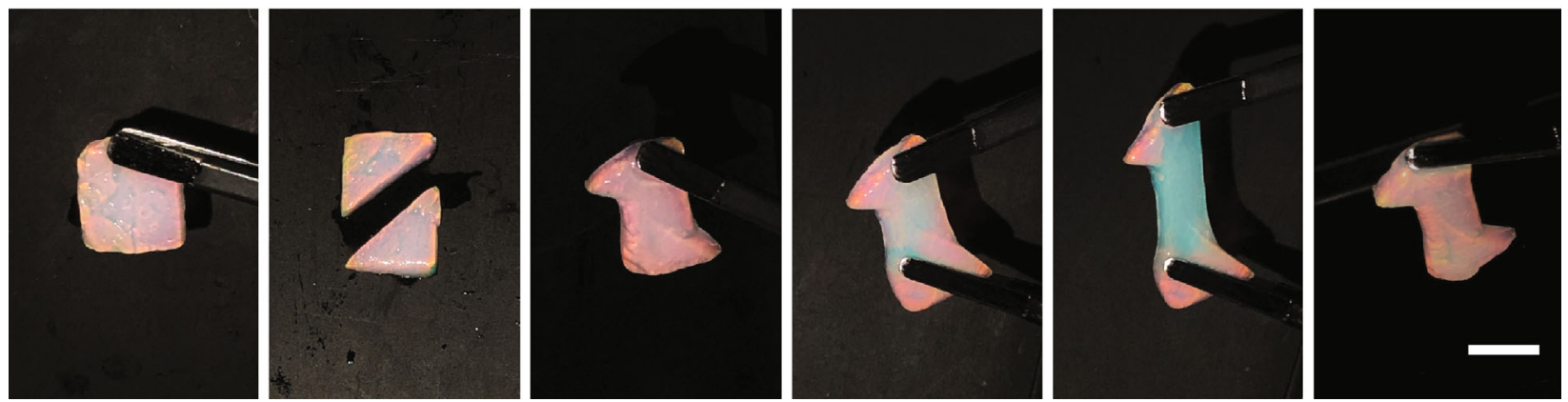

(c)

FIgURe 3: Visual demonstration of self-healing properties of the three colloidal PC hydrogels with PBA-microgels at different diameters against physical damage. Hydrogel sizes: $1.5 \mathrm{~cm} \times 1.5 \mathrm{~cm}$. (a) Blue PC hydrogel; (b) green PC hydrogel; (c) red PC hydrogel. Scale bar $=1 \mathrm{~cm}$.

subjected to a stretching force. In addition, no significant difference was observed on the reflection spectra of the repaired sections when the stretching force was removed. Interestingly, we found that the external tension applied on the colloidal PC hydrogels all led to a blue shift in the structural colors (Figure 3), mainly due to the decreased interplanar distance of the diffracting plane under tension, indicating one of the potential applications of our PC hydrogels as a mechanical sensor [44]. Nevertheless, the hydrogels could recover their original structural colors once the tension forces were removed and the hydrogels shrunk to their original sizes. In addition, we found our colloidal PC hydrogels could be stretched up to $155 \%$ of the original length, indicating good flexibility to resist an external tensile force (Figure S4). These phenomena indicated that our PC hydrogels possessed self-reparability and reversibility in the structural colors.

To further demonstrate the self-recovery, rheology examination of the colloidal PC hydrogels was carried out.
Dynamic oscillatory frequency sweeps were first studied with frequencies $(\omega)$ ranging from 0.1 to $100 \mathrm{rad} \mathrm{s}^{-1}$ (Figure 4(a)). All the hydrogels showed frequency dependence viscoelastic behaviors with a higher storage modulus $\left(G^{\prime}\right)$ than the loss modulus $\left(G^{\prime \prime}\right)$ across the whole range of frequencies tested, suggesting their dynamic and gel-like characteristics [56, 57]. Strain sweep tests were then performed to find the critical strain values required to damage the gel networks. As shown in Figure 4(b), the $G^{\prime}$ and $G^{\prime \prime}$ of all three hydrogels kept constant until the strain $(\gamma)$ reached up to $100 \%$, indicating good resistance for mechanical agitations. However, a dramatic decrease was observed for both $G^{\prime}$ and $G^{\prime \prime}$ when the strain was further increased. This is resulted from the severe breakage of dynamic PBA-cis-diol interactions and collapse of the hydrogel networks, showing a transition of the colloidal PC hydrogels from gel to sol state [49]. The self-recovery of the hydrogels after network failure under high strains was finally investigated using a step-strain sweep with alternate low (1\%) and high (150\%) strains. As shown in 

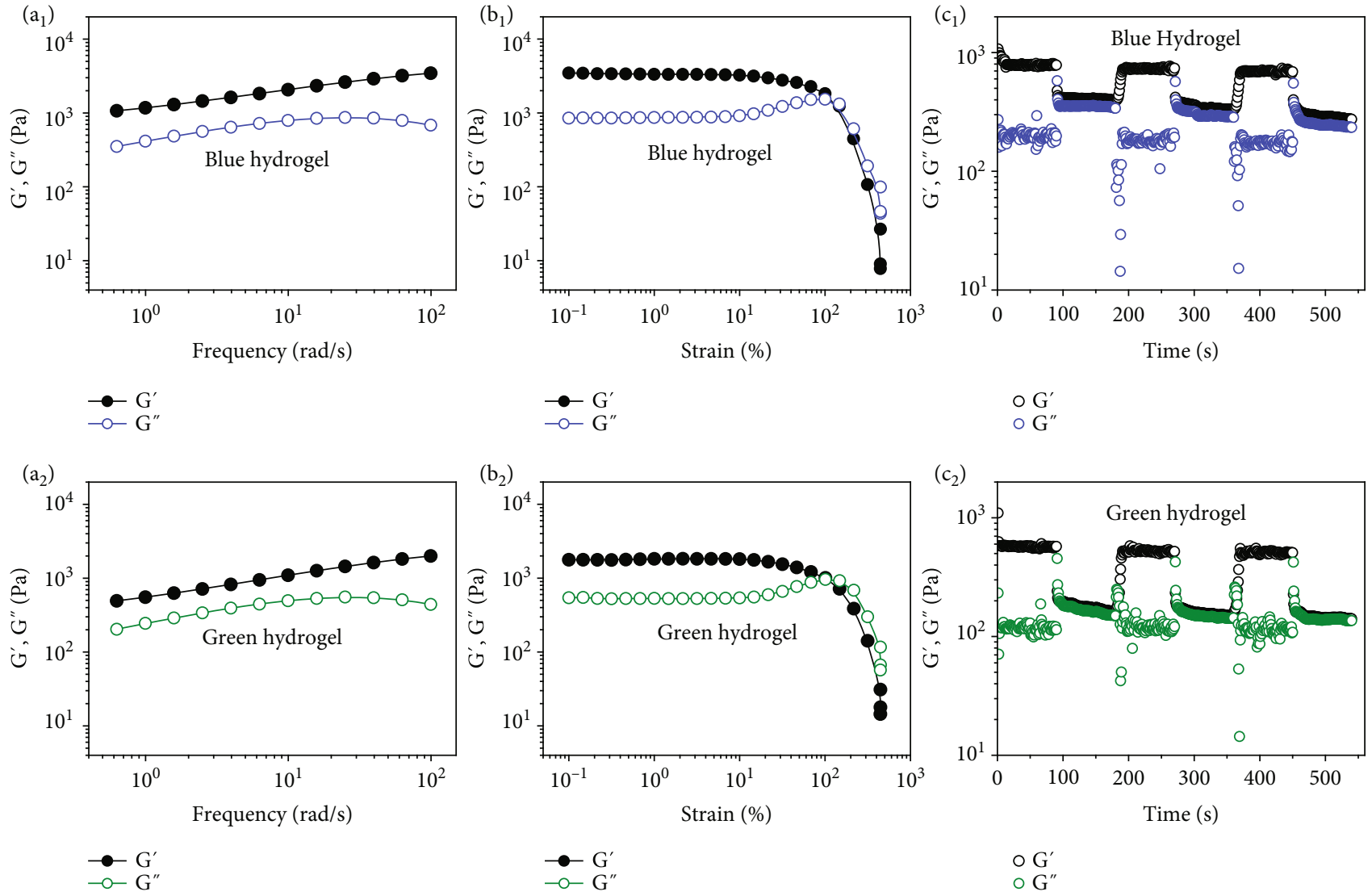

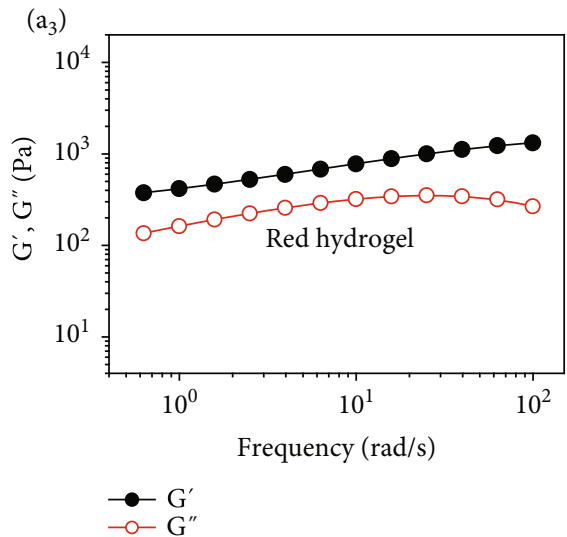

(a)

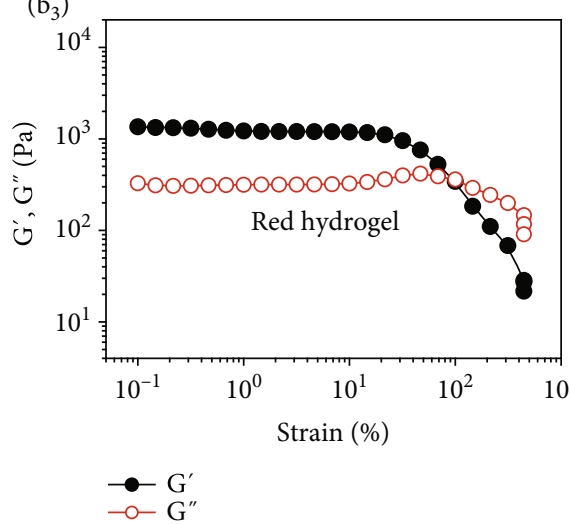

(b)

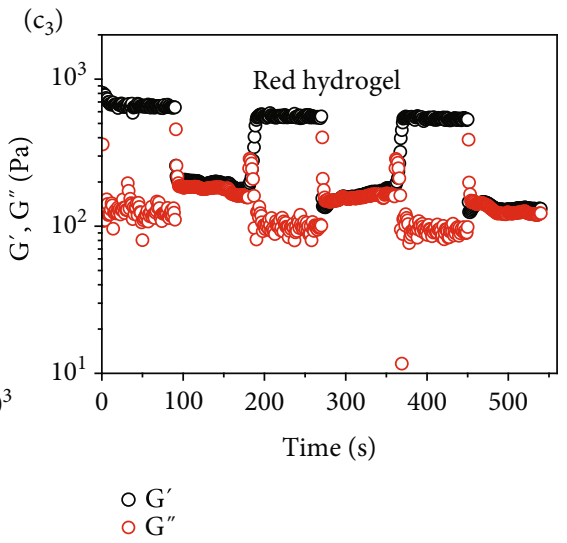

(c)

FIGURE 4: (a) Dynamic oscillatory frequency sweeps $(\gamma=1 \%)$, (b) strain amplitude sweeps $\left(\omega=1 \mathrm{rad} \mathrm{s}^{-1}\right)$, and (c) step-strain sweeps $(\gamma=1 \%$ or $150 \%, \omega=1 \mathrm{rad} \mathrm{s}^{-1}$ ) of the dynamic colloidal PC hydrogels at $15^{\circ} \mathrm{C} . \mathrm{G}^{\prime}$ : storage modulus; $\mathrm{G}^{\prime \prime}$ : loss modulus.

Figure 4(c), the mechanical properties of all three colloidal PC hydrogels could immediately recover as soon as the highoscillation excitation was removed. Moreover, repeated dynamic strain sweep revealed that both $G^{\prime}$ and $G^{\prime \prime}$ could get back to their initial values without significant loss, demonstrating the robust self-healing property of our PC hydrogels and fast recovery of the hydrogel networks after physical damage. This result, together with the self-reparability of structural colors, jointly demonstrated the perfect selfrecovery of our PC hydrogels in both structure and function.

In addition to self-recovery, another remarkable characteristic of PC hydrogels is the diversity of coloration derived from combinatorial structural colors. To demonstrate the versatility of our self-healing colloidal PC hydrogels for mimicking the multistructural colors of natural PC materials, three pieces of hydrogels with different colors were used for conceptual construction of artificial PC materials with combinatorial structural colors (Figure 5). Similar to the selfhealing mechanism through the formation of interfacial PBA-cis-diol interactions, the red, green, and blue colloidal PC hydrogels could easily assemble together with stable mechanical strength, resulting in a hybrid colloidal PC hydrogel displaying three combinatorial structural colors (Figure 5(a)). In addition to the multistructural assembly in 

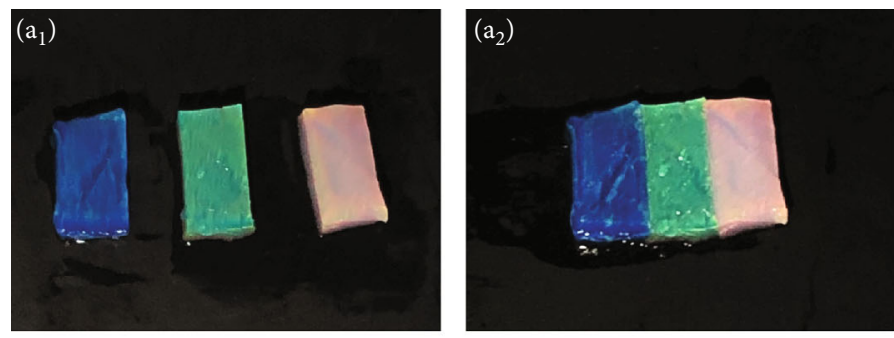

(a)
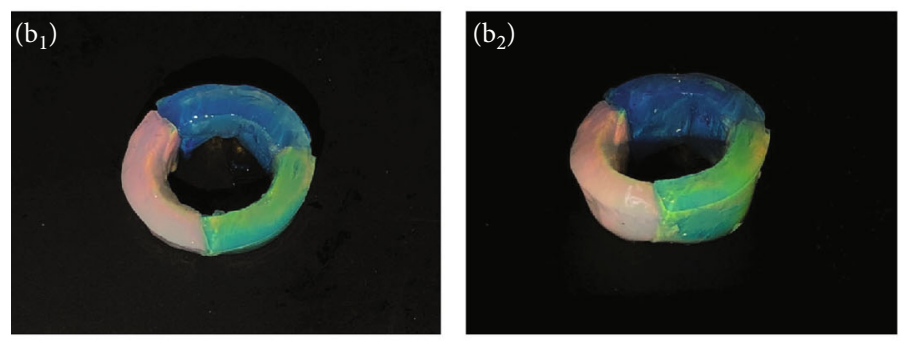

(b)
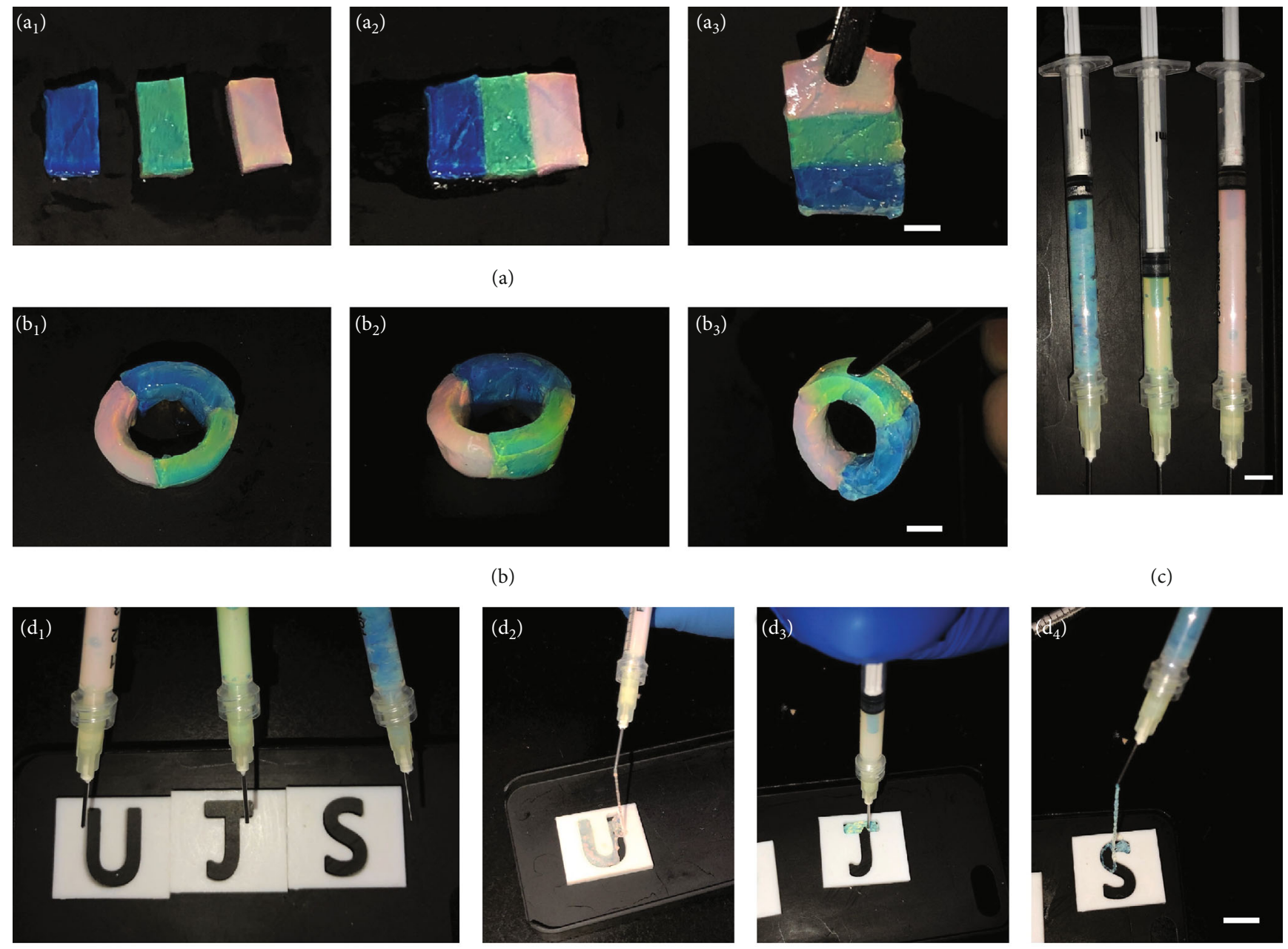

(d)
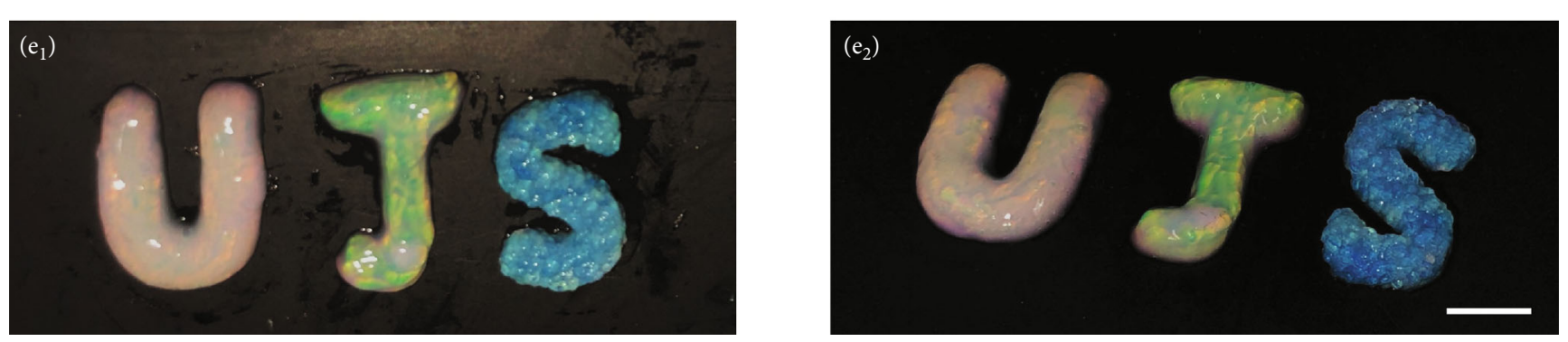

(e)

FIgURE 5: (a) The assembled hybrid colloidal PC hydrogels with three combinatorial structure colors at $2 \mathrm{D}$ plane. Hydrogel sizes: $1.5 \mathrm{~cm}$ $\times 3.0 \mathrm{~cm}$. Scale bar $=1 \mathrm{~cm}$. (b) The assembled 3D curved colloidal PC hydrogel ring. Scale bar $=1 \mathrm{~cm}$. (c) The three dynamic colloidal PC hydrogels could be easily transferred into syringes. Scale bar $=1 \mathrm{~cm}$. (d) The injection of dynamic colloidal PC hydrogels for the construction of photonic letter patterns. Teflon molds: $3.0 \mathrm{~cm} \times 3.0 \mathrm{~cm}$. Scale bar $=1 \mathrm{~cm}$. (e) The photos of photonic letter patterns shot from different angles $\left(90^{\circ}\right.$ and $\left.45^{\circ}\right)$. Scale bar $=1 \mathrm{~cm}$.

the $2 \mathrm{D}$ plane, the PC hydrogels were also used to construct a $3 \mathrm{D}$ curved hydrogel ring. As shown in Figure 5(b), a threecolor PC hydrogel ring could be readily obtained, implying the possibility of our dynamic colloidal PC hydrogels for precise construction of PC devices with complex macrostructures and multiple structural colors.

Apart from the versatility in multistructural assembly, the mechanical agitation-induced gel-to-sol transition also implied the possibility of our colloidal PC hydrogels for injection. Since the disruption of dynamic crosslinks by shear stress would provide broken segments with higher mobility within the hydrogel matrix, the shear-thinning behaviors were visually demonstrated by injecting our colloidal PC hydrogels through a needle $(21 \mathrm{G})$. The hydrogels could be easily placed into a syringe and then applied with pressure for injection. As shown in Figures 5(c) and 5(d) and Movie 
S1, all the hydrogels showed bright structural color due to the formation of ordered structure via reversible phenylboronate covalent bond before injection. These hydrogels could be favorably injected through the syringe followed by a rapid recovery of the structural colors. By using predesigned Teflon molds with letter patterns, three hydrogel letters with different structural colors could be constructed (Figure 5(e)). In addition, the three injected PC hydrogel letters all showed bright colors from different angels, i.e., angle-independent structural colors. Next, we performed the extrusion-based 3D printing test to demonstrate the printability of our colloidal PC hydrogels. As shown in Figure S5, a multilayered 3D scaffold with a fiber diameter of $\sim 500 \mu \mathrm{m}$ was obtained using blue and green PC hydrogels as the inks, which exhibited high printing resolution. Conceivably, the injectability, moldability, and printability of our dynamic PC hydrogels reflected a very attractive potential in the development of diversified artificial PC materials or scaffolds for different biomedical applications.

\section{Conclusion}

In summary, we propose here a reversible boronate covalent strategy for the design of dynamic colloidal PC hydrogels with self-healing and injectable properties. The motivation of this study is to structurally and functionally mimic the dynamic characteristic and complexity of natural PC materials (e.g., self-recovery and diversity of biogenic periodic nanostructures.). Through reversible crosslinking of adjacent particles in self-assembled microgel colloidal crystals, the dynamic colloidal PC hydrogels could be tactfully constructed. As proof of concept, three types of hydrogels with different microgel sizes and structural colors were prepared in this work. Due to the reversible covalent interactions within the microgel colloidal crystals, the resultant PC hydrogels showed perfect self-healing ability against physical damage and rapid recovery of the structural colors after injection. These properties suggested the superiority of our dynamic colloidal PC hydrogels for the construction of artificial PC materials with complex macrostructures, multiple structural colors, and strong mechanical stress tolerance. We believe that our dynamic colloidal PC hydrogels will be highly promising for different applications, such as environment monitoring, anticounterfeiting, optical devices, energy conversion, and biomedical engineering. Overall, in addition to the great significance in functional biomimicry of natural PC materials, our work also presents an available easy-toperform strategy for the development of diversified artificial PC materials or devices.

\section{Materials and Methods}

4.1. Materials. The solvents deuterium oxide $\left(\mathrm{D}_{2} \mathrm{O}\right)$ and dimethyl sulfoxide- $\mathrm{d}_{6}\left(\mathrm{DMSO}_{6} \mathrm{~d}_{6}\right.$ ) (99.5 atom \% D, SigmaAldrich) for ${ }^{1} \mathrm{H}$ NMR spectroscopy, $N$-isopropylacrylamide (NIPAAm, 98\%, Macklin), crosslinker $N, N{ }^{\prime}$-methylenebis(acrylamide) (BIS, 99\%, Aladdin), 4-carboxy-3-fluorophenylboronic acid (CFPBA, 98\%, J\&K), sodium dodecyl sulfate (SDS, 98\%, Aladdin), photo initiator 2-hydroxy-4' -(2-hydroxyethoxy)-2-methylpropiophenone (HMP, 98\%, Aladdin), and potassium persulfate (KPS, 99.5\%, Sinopharm) were used as received. Dichloromethane (DCM), triethylamine (TEA), tetrahydrofuran (THF), and N,N-dimethylformamide (DMF) for chemical synthesis were dried with $\mathrm{CaH}_{2}$ and distilled by a general method before use. Other reagents and AR-grade solvents in this work were purchased from Shanghai Reagent General Factory and used as received. Milli-Q water was purified with a Thermo Scientific Barnstead NANOpure Diamond Water Purification Systems to give a minimum resistivity of $18.2 \mathrm{M} \Omega \cdot \mathrm{cm}$. The phenylboronic acid (PBA) monomer 4-(2-acrylamidoethylcarbamoyl)-3-fluorophenylboronic acid (AFPBA) and glycomonomer 3-gluconamidopropyl methacrylamide (GAPMA) were synthesized as follows.

4.2. Synthesis of Phenylboronic Acid Monomer (AFPBA). The phenylboronic acid monomer 4-(2-acrylamidoethylcarbamoyl)-3-fluorophenylboronic acid (AFPBA) was synthesized according to a previously reported method [51]. 4-Carbamoyl-3-fluorophenylboronic acid (CFPBA) (5 g, $27.2 \mathrm{mmol}$ ) was added to $50 \mathrm{~mL}$ of thionyl chloride and refluxed for $6 \mathrm{~h}$ under an argon atmosphere. After evaporation in vacuum, the residual solid was mixed with $\mathrm{N}$-carboxybenzoxy-1,2diaminoethane hydrochloride $(9.4 \mathrm{~g}, 40.8 \mathrm{mmol})$ in $\mathrm{THF}$ $(90 \mathrm{~mL})$, to which $28 \mathrm{~mL}$ of trimethylamine was slowly added in an ice-cooled bath, stirring at ambient temperature for $6 \mathrm{~h}$. The resulting solution was washed with diluted hydrochloric acid brine, and the organic layer was evaporated to obtain a white solid. It was then dissolved in $400 \mathrm{~mL}$ of ethanol and was hydrogenized in the presence of $10 \% \mathrm{Pd} / \mathrm{C}(1.0 \mathrm{~g})$ at $40^{\circ} \mathrm{C}$ for $3 \mathrm{~h}$. The mixture was then filtrated and evaporated using a rotary evaporator. The obtained white solid was dried and dissolved in $150 \mathrm{~mL}$ of sodium carbonate aqueous solution (100 mM, pH 10). Acryloyl chloride (4.0 mL, $50 \mathrm{mmol}$ ) was added in an ice-cooled bath at weakly basic $\mathrm{pH}$ using $\mathrm{NaOH}$ aqueous solution. After stirring at ambient temperature for $6 \mathrm{~h}$, the mixture was acidified using $\mathrm{HCl}$ solution, and the resulting AFPBA was recrystallized in acetone. The obtained crystal was filtrated, washed with acetone, and finally left to dry in a vacuum. (45\% in yield): the chemical structure was characterized by ${ }^{1} \mathrm{H}$ NMR and LC-MS.

${ }^{1} \mathrm{H}$ NMR (300 MHz, d6-DMSO, Figure S1): $\delta 8.37$ (s, $2 \mathrm{H}$, $\left.\mathrm{B}(\mathrm{OH})_{2}\right) ; \delta 8.23 \sim 8.24$ (m, 1H, -CO-NH-); $\delta 7.55 \sim 7.65(3 \mathrm{H}$, Ar-H); $\delta 6.07 \sim 6.21\left(\mathrm{~m}, 2 \mathrm{H},-\mathrm{CH}=\mathrm{CH}_{2}\right) ; \delta 5.58 \sim 5.60(\mathrm{~d}, 1 \mathrm{H}$, $\left.-\mathrm{CH}=\mathrm{CH}_{2}\right) ; \delta$ 2.30 3.33 (m, 4H, $\left.-\mathrm{NHCH}_{2} \mathrm{CH}_{2} \mathrm{NH}-\right)$; LCMS (Figure S1): $280.32[\mathrm{M}] ; 281.31[\mathrm{M}+\mathrm{H}]^{+} ; 303.14[\mathrm{M}$ $+\mathrm{Na}]^{+}$.

4.3. Synthesis of the Glycomonomer (GAPMA). GAPMA glycomonomer was prepared according to a previous method. D-gluconolactone $(20 \mathrm{~g}, 112.3 \mathrm{mmol})$ and APMA (25 g, $140.4 \mathrm{mmol}$ ) were dissolved in $400 \mathrm{~mL}$ methanol. Then, the solution was added with triethylamine $(20 \mathrm{~mL}, 142.8 \mathrm{mmol})$. The mixture was stirred overnight at $25^{\circ} \mathrm{C}$. The resulting mixture was then filtered with a G5 funnel and then concentrated under vacuum and precipitated out in methanol at $-20^{\circ} \mathrm{C}$. The crude product was dissolved in DMF and recovered by a large excess amount of acetone. The obtained white 
solid was dried in vacuo (23.3 g, 65.4\% in yield): the chemical structure was characterized by ${ }^{1} \mathrm{H}$ NMR.

${ }^{1} \mathrm{H}$ NMR (400 MHz, D2O, Figure S2) $\delta 5.62(\mathrm{~s}, 1 \mathrm{H}), \delta 5.37$ $(\mathrm{s}, 1 \mathrm{H}), \delta 4.24 \sim 4.23(\mathrm{~d}, 1 \mathrm{H}), \delta 4.01(\mathrm{t}, 1 \mathrm{H}), \delta 3.54 \sim 3.75(\mathrm{~m}, 4 \mathrm{H})$, $\delta 3.24 \sim 3.21(\mathrm{t}, 4 \mathrm{H}), \delta 1.85(\mathrm{~s}, 3 \mathrm{H}), \delta 1.68 \sim 1.72(\mathrm{q}, 2 \mathrm{H})$.

4.4. Preparation of PBA-Containing Microgels (PBAMicrogels). The typical procedure for the synthesis of the phenylboronic acid-containing microgels (PBA-microgel-S) is presented as follows: NIPAAm (740 mg), BIS (22 mg), and SDS (12 mg) were added into a three-neck roundbottom flask $(100 \mathrm{~mL})$ and then $50 \mathrm{~mL}$ of deionized water was put into the flask. A clear solution was obtained after ultrasonication and purged with $\mathrm{N}_{2}$ for $30 \mathrm{~min}$ at room temperature. Then, the flask was immersed into a thermostatic water bath at $35^{\circ} \mathrm{C}$ with $\mathrm{N}_{2}$ purged continually. AFPBA (78 mg, dissolved in $0.5 \mathrm{~mL}$ of DMF) was transferred to the flask. After the solution was heated to $70^{\circ} \mathrm{C}$, KPS $(40 \mathrm{mg}$, dissolved in $2.5 \mathrm{~mL}$ of deionized water) was dropped to initiate the reaction. The polymerization lasted for $5 \mathrm{~h}$ at 70 with $\mathrm{N}_{2}$ condition. After the reaction, the white suspension was purified by dialysis against water with frequent water change for 2 weeks, lyophilized, and stored in a refrigerator for further use (entry 1 in Table S1).

PBA-microgel-M and PBA-microgel-L were synthesized and purified under the identical conditions except for changing the ratio of the reagents (entries 2 and 3 in Table S1).

4.5. Preparation of Dynamic Colloid Photonic Crystal Hydrogels. The typical procedure for the synthesis of dynamic colloidal PC hydrogel (blue hydrogel) is presented as follows: photoinitiator HHMP (6.6 mg), glycomonomer GAPMA (40 mg), and crosslinker BIS (2 mg) were dissolved in $1 \mathrm{~mL}$ of water solution ( $\mathrm{pH} 7.5)$. A clear solution was obtained after ultrasonication, and then the PBA-microgel$\mathrm{S}(128 \mathrm{mg})$ was transferred to the monomer solution. After swelling $12 \mathrm{~h}$ in the dark, a blue colloid crystal was obtained, and then it was transferred into a $10 \times 10 \times 3 \mathrm{~mm}$ mold. The system was then photopolymerized by exposure to UV light at $15^{\circ} \mathrm{C}$ for $24 \mathrm{~h}(\lambda=365 \mathrm{~nm})$. The resulting films were released from the device and stored in a hermetical vessel at $4^{\circ} \mathrm{C}$ for further use (entry 4 in Table S2).

The green and red PC hydrogels (green hydrogel and red hydrogel) were synthesized similarly (entries 5 and 6 in Table S2).

4.6. Characterization. The structures of two synthesized monomers (AFPBA and GAPMA) were measured by ${ }^{1} \mathrm{H}$ NMR (Varian Unity plus-400 spectrometer, $400 \mathrm{MHz}$ ) using $\mathrm{D}_{2} \mathrm{O}$ or DMSO as solvent. The morphologies of the dry microgels were characterized with a SEM. DLS study was performed to obtain their sizes and particle dispersion indices (PDIs) in water. A DLS spectrometer (ZETAPALS/BI-200SM) with a detector (CROSS-PMT) was utilized for this purpose. The scattered light of a vertically polarized He-Ne laser $(659 \mathrm{~nm})$ was measured at an angle of $90^{\circ}$ at room temperature. The compositions of all the nanoparticles and color colloid crystals were obtained by Fourier transform infrared spectroscopy (FT-IR, Nicolet
Magna-560 FTI spectrometer). Reflection spectra of the PC materials were measured with a fiber optic spectrometer (AvaSpec-2048). Rheology examination of the colloidal PC hydrogels was carried out on an Anton Paar MCR301 rheometer at $15^{\circ} \mathrm{C}$ with a $25 \mathrm{~mm}$ diameter flat plate attached to a transducer. In addition, we performed tensile tests to characterize the mechanical properties of the green PC hydrogel through a universal mechanical testing system (Instron, US). Specimens with dimensions of $20 \times 5$ $\times 1.5 \mathrm{~mm}$ (length $\times$ width $\times$ height) were loaded onto the testing machine, and the tensile test was performed at room temperature with a speed of $1 \mathrm{~mm} / \mathrm{min}$ until specimen failure. We also evaluated the $3 \mathrm{D}$ printability of our dynamic PC hydrogels. The pregel solution of the PC hydrogel was printed by extrusion-based $3 \mathrm{D}$ printer (Panowin F1) through a $20 \mathrm{G}$ plastic-steel flat tip needle with the aid of 3DMax software. The printing pump was set at $4^{\circ} \mathrm{C}$ and a moving speed of $0.35 \mathrm{~mm} / \mathrm{s}$.

\section{Data Availability}

The data that support the findings of this study are available from the corresponding author on request.

\section{Conflicts of Interest}

The authors declare that there is no conflict of interest regarding the publication of this article.

\section{Authors' Contributions}

Y. Ma and P. He contributed equally to this work. Y. Ma and P. He performed most chemical syntheses, material preparation, and characterizations. X. Chen, W. Xie, W. Yin, Q. Zhang, and M. Wang partly performed material characterization. Y. Ma and G. Pan conceived and supervised the study and planned the experiments. Y. Ma, P. He, X. Zhao, and G. Pan analyzed the data and wrote the manuscript with help from all authors.

\section{Acknowledgments}

We acknowledge the financial support from the National Key Research and Development Program of China (2019YFA0112000), the National Natural Science Foundation of China (21875092, 21706099, and 91649204), the start-up fund (1-ZE7S) and central research fund (G-YBWS) from the Hong Kong Polytechnic University, the China Postdoctoral Science Foundation funded project (2018M642174), the Postdoctoral Science Foundation of Jiangsu Province (2019K145), the Natural Science Foundation of Jiangsu Province (BK20160056 and BK20160491), the Innovation and Entrepreneurship Program of Jiangsu Province, and the Six Talent Peaks Project in Jiangsu Province (2018XCL-013).

\section{Supplementary Materials}

Supplementary 1. Movie S1: the injectability of dynamic colloidal photonic hydrogels. 
Supplementary 2. Table S1: the ratio of NIPAAm, BIS, SDS, and AMCFPBA in the precipitation polymerization. Table S2: the parameters for PC hydrogel construction. Figure S1: $1 \mathrm{H}$ NMR and ESI-MS of 4-(2-acrylamidoethylcarbamoyl)3-fluorophenylboronic acid (AFPBA). Figure S2: 1H NMR spectra for 3-gluconamidopropyl methacrylamide (GAPMA) monomer in D2O. Figure S3: the enlarged images of selfhealing process of the colloidal PC hydrogels. Figure S4: the stress-strain curve of tensile test of green colloidal PC hydrogel. Figure S5: 3D printability of the blue and green PC hydrogels (scale bar $=5 \mathrm{~mm}$ ).

\section{References}

[1] A. R. Parker, V. L. Welch, D. Driver, and N. Martini, "Structural colour: opal analogue discovered in a weevil," Nature, vol. 426, no. 6968, pp. 786-787, 2003.

[2] A. G. Richards Jr. and T. F. Anderson, "Electron microscope studies of insect cuticle, with a discussion of the application of electron optics to this problem," Journal of Morphology, vol. 71, no. 1, pp. 135-183, 1942.

[3] S. Kinoshita and S. Yoshioka, "Structural colors in nature: the role of regularity and irregularity in the structure," ChemPhysChem, vol. 6, no. 8, pp. 1442-1459, 2005.

[4] Y. Zhao, "Structural color materials in evolution," Materials Today, vol. 19, pp. 420-421, 2016.

[5] Y. Zhao, L. Shang, Y. Cheng, and Z. Gu, "Spherical colloidal photonic crystals," Accounts of Chemical Research, vol. 47, no. 12, pp. 3632-3642, 2014.

[6] Y. Zhao, Z. Xie, H. Gu, C. Zhu, and Z. Gu, "Bio-inspired variable structural color materials," Chemical Society Reviews, vol. 41, no. 8, pp. 3297-3317, 2012.

[7] S.-H. Kim, S.-J. Jeon, G.-R. Yi, C.-J. Heo, J. H. Choi, and S.M. Yang, "Optofluidic assembly of colloidal photonic crystals with controlled sizes, shapes, and structures," Advanced Materials, vol. 20, no. 9, pp. 1649-1655, 2008.

[8] J. Hou, M. Li, and Y. Song, "Patterned colloidal photonic crystals," Angewandte Chemie, International Edition, vol. 57, no. 10, pp. 2544-2553, 2018.

[9] Y. Fu, C. A. Tippets, E. U. Donev, and R. Lopez, "Structural colors: from natural to artificial systems," Wiley Interdisciplinary Reviews: Nanomedicine and Nanobiotechnology, vol. 8, no. 5, pp. 758-775, 2016.

[10] A. Fang, Z. Q. Zhang, S. G. Louie, and C. T. Chan, "Pseudospin-1 physics of photonic crystals," Research, vol. 2019, article 3054062, 15 pages, 2019.

[11] H. Inan, M. Poyraz, F. Inci et al., "Photonic crystals: emerging biosensors and their promise for point-of-care applications," Chemical Society Reviews, vol. 46, no. 2, pp. 366-388, 2017.

[12] B. Ye, H. Ding, Y. Cheng et al., "Photonic crystal microcapsules for label-free multiplex detection," Advanced Materials, vol. 26, no. 20, pp. 3270-3274, 2014.

[13] M. Qin, Y. Huang, Y. Li et al., "A rainbow structural-color chip for multisaccharide recognition," Angewandte Chemie, International Edition, vol. 55, no. 24, pp. 6911-6914, 2016.

[14] S. H. Kim, J. W. Shim, and S. M. Yang, "Microfluidic multicolor encoding of microspheres with nanoscopic surface complexity for multiplex immunoassays," Angewandte Chemie, International Edition, vol. 50, no. 5, pp. 1171-1174, 2011.
[15] Y. Huang, F. Li, M. Qin, L. Jiang, and Y. Song, "A multistopband photonic-crystal microchip for high-performance metal-ion recognition based on fluorescent detection," Angewandte Chemie, International Edition, vol. 52, no. 28, pp. 7296-7299, 2013.

[16] H. Wang, X. Gu, R. Hu, J. W. Lam, D. Zhang, and B. Z. Tang, "Luminescent photonic crystals with multi-functionality and tunability," Chemical Science, vol. 7, no. 9, pp. 5692-5698, 2016.

[17] X. Sun, J. Zhang, X. Lu, X. Fang, and H. Peng, "Mechanochromic photonic-crystal fibers based on continuous sheets of aligned carbon nanotubes," Angewandte Chemie, International Edition, vol. 54, no. 12, pp. 3630-3634, 2015.

[18] M. Elsherif, M. U. Hassan, A. K. Yetisen, and H. Butt, "Wearable contact lens biosensors for continuous glucose monitoring using smartphones," ACS Nano, vol. 12, no. 6, pp. $5452-$ 5462,2018

[19] F. Hu, L. Zhang, W. Liu, X. Guo, L. Shi, and X. Y. Liu, "Gelbased artificial photonic skin to sense a gentle touch by reflection," ACS Applied Materials \& Interfaces, vol. 11, no. 17, pp. 15195-15200, 2019.

[20] H. Gu, Y. Zhao, Y. Cheng et al., "Tailoring colloidal photonic crystals with wide viewing angles," Small, vol. 9, no. 13, pp. 2266-2271, 2013.

[21] C. Liu, H. Ding, Z. Wu et al., "Tunable structural color surfaces with visually self-reporting wettability," Advanced Functional Materials, vol. 26, no. 43, pp. 7937-7942, 2016.

[22] C. Zhu, W. Xu, L. Chen, W. Zhang, H. Xu, and Z. Z. Gu, "Magnetochromatic microcapsule arrays for displays," Advanced Functional Materials., vol. 21, no. 11, pp. 2043-2048, 2011.

[23] J. S. Lee, K. Je, and S. H. Kim, "Designing multicolored photonic micropatterns through the regioselective thermal compression of inverse opals," Advanced Functional Materials., vol. 26, no. 25, pp. 4587-4594, 2016.

[24] W. Wang, X. Fan, F. Li et al., "Magnetochromic photonic hydrogel for an alternating magnetic field-responsive color display," Advanced Optical Materials., vol. 6, no. 4, p. 1701093, 2018.

[25] H. S. Lee, J. H. Kim, J. S. Lee et al., "Magnetoresponsive discoidal photonic crystals toward active color pigments," Advanced Materials, vol. 26, no. 33, pp. 5801-5807, 2014.

[26] X. Zhang, Y. Liu, S.-T. Lee, S. Yang, and Z. Kang, "Coupling surface plasmon resonance of gold nanoparticles with slowphoton-effect of $\mathrm{TiO}_{2}$ photonic crystals for synergistically enhanced photoelectrochemical water splitting," Energy \& Environmental Science., vol. 7, no. 4, pp. 1409-1419, 2014.

[27] Q. Yang, M. Li, J. Liu et al., "Hierarchical $\mathrm{TiO}_{2}$ photonic crystal spheres prepared by spray drying for highly efficient photocatalysis," Journal of Materials Chemistry A., vol. 1, no. 3, pp. 541-547, 2013.

[28] M. Wang, L. He, W. Xu, X. Wang, and Y. Yin, "Magnetic assembly and field-tuning of ellipsoidal-nanoparticle-based colloidal photonic crystals," Angewandte Chemie, International Edition, vol. 54, no. 24, pp. 7077-7081, 2015.

[29] J. Yang, M. K. Choi, D.-H. Kim, and T. Hyeon, "Designed assembly and integration of colloidal nanocrystals for device applications," Advanced Materials, vol. 28, no. 6, pp. 1176$1207,2016$.

[30] Q. Fu, H. Zhu, and J. Ge, "Electrically tunable liquid photonic crystals with large dielectric contrast and highly saturated 
structural colors," Advanced Functional Materials, vol. 28, no. 43, p. $1804628,2018$.

[31] J. Wang, Y. Zhang, S. Wang, Y. Song, and L. Jiang, "Bioinspired colloidal photonic crystals with controllable wettability," Accounts of Chemical Research, vol. 44, no. 6, pp. 405415, 2011.

[32] C. Shao, Y. Liu, J. Chi, J. Wang, Z. Zhao, and Y. Zhao, "Responsive inverse opal scaffolds with biomimetic enrichment capability for cell culture," Research, vol. 2019, article 9783793, 10 pages, 2019.

[33] Y. Wang, S. E. Naleway, and B. Wang, "Biological and bioinspired materials: structure leading to functional and mechanical performance," Bioactive Materials, vol. 5, no. 4, pp. 745$757,2020$.

[34] Y. Liu, Y. Wang, Y. Wang, Y. Shu, H. Tan, and Y. Zhao, "Bioinspired structural color particles with multi-layer graphene oxide encapsulated nanoparticle components," Bioactive Materials, vol. 5, no. 4, pp. 917-923, 2020.

[35] X. Chen, Q. Guo, W. Chen et al., "Biomimetic design of photonic materials for biomedical applications," Acta Biomaterialia, vol. 121, pp. 143-179, 2021.

[36] J. Y. Wang, Y. Cao, Y. Feng, F. Yin, and J. P. Gao, "Multiresponsive inverse-opal hydrogels," Advanced Materials, vol. 19, no. 22, pp. 3865-3871, 2007.

[37] M. Honda, T. Seki, and Y. Takeoka, "Dual tuning of the photonic band-gap structure in soft photonic crystals," Advanced Materials, vol. 21, no. 18, pp. 1801-1804, 2009.

[38] C. I. Aguirre, E. Reguera, and A. Stein, "Tunable colors in opals and inverse opal photonic crystals," Advanced Functional Materials, vol. 20, no. 16, pp. 2565-2578, 2010.

[39] X. Hu, Z. Ma, J. Li et al., "Superior water anchoring hydrogel validated by colorimetric sensing," Materials Horizons, vol. 7, no. 12, pp. 3250-3257, 2020.

[40] J. H. Holtz and S. A. Asher, "Polymerized colloidal crystal hydrogel films as intelligent chemical sensing materials," Nature, vol. 389, no. 6653, pp. 829-832, 1997.

[41] C. E. Reese, A. V. Mikhonin, M. Kamenjicki, A. Tikhonov, and S. A. Asher, "Nanogel nanosecond photonic crystal optical switching," Journal of the American Chemical Society, vol. 126, no. 5, pp. 1493-1496, 2004.

[42] M. Chen, L. Zhou, Y. Guan, and Y. Zhang, "Polymerized microgel colloidal crystals: photonic hydrogels with tunable band gaps and fast response rates," Angewandte Chemie, International Edition, vol. 52, no. 38, pp. 9961-9965, 2013.

[43] J. Chen, L. Xu, M. Yang, X. Chen, X. Chen, and W. Hong, "Highly stretchable photonic crystal hydrogels for a sensitive mechanochromic sensor and direct ink writing," Chemistry of Materials, vol. 31, no. 21, pp. 8918-8926, 2019.

[44] F. Fu, Z. Chen, Z. Zhao et al., "Bio-inspired self-healing structural color hydrogel," Proceedings of the National Academy of Sciences of the United States of America, vol. 114, no. 23, pp. 5900-5905, 2017.

[45] Z. Zhu, J. D. Liu, C. Liu et al., "Microfluidics-assisted assembly of injectable photonic hydrogels toward reflective cooling," Small, vol. 16, no. 9, article 1903939, 2019.

[46] J. Zhang, Y. Li, X. Zhang, and B. Yang, "Colloidal self-assembly meets nanofabrication: from two-dimensional colloidal crystals to nanostructure arrays," Advanced Materials, vol. 22, no. 38, pp. 4249-4269, 2010.

[47] S.-N. Yin, J. Liu, D. Wu, S. Chen, and W. Xia, "Robust selfhealing magnetically induced colloidal photonic crystal hydro- gels," ACS Applied Polymer Materials, vol. 2, no. 2, pp. 448 454, 2019.

[48] G. Pan, B. Guo, Y. Ma et al., "Dynamic introduction of cell adhesive factor via reversible multicovalent phenylboronic acid/cis-diol polymeric complexes," Journal of the American Chemical Society, vol. 136, no. 17, pp. 6203-6206, 2014.

[49] V. Yesilyurt, M. J. Webber, E. A. Appel, C. Godwin, R. Langer, and D. G. Anderson, "Injectable self-healing glucoseresponsive hydrogels with $\mathrm{pH}$-regulated mechanical properties," Advanced Materials, vol. 28, no. 1, pp. 86-91, 2016.

[50] Y. Ma, X. Tian, L. Liu, J. Pan, and G. Pan, "Dynamic synthetic biointerfaces: from reversible chemical interactions to tunable biological effects," Accounts of Chemical Research., vol. 52, no. 6, pp. 1611-1622, 2019.

[51] G. Pan, Q. Guo, C. Cao, H. Yang, and B. Li, “Thermo-responsive molecularly imprinted nanogels for specific recognition and controlled release of proteins," Soft Matter, vol. 9, no. 14, pp. 3840-3850, 2013.

[52] M. Andersson and S. L. Maunu, "Structural studies of poly (Nisopropylacrylamide) microgels: effect of SDS surfactant concentration in the microgel synthesis," Journal of Polymer Science: Polymer Physics, vol. 44, no. 23, pp. 3305-3314, 2006.

[53] G. Pan, Q. Guo, Y. Ma, H. Yang, and B. Li, "Thermo-responsive hydrogel layers imprinted with RGDS peptide: a system for harvesting cell sheets," Angewandte Chemie International Edition, vol. 52, no. 27, pp. 6907-6911, 2013.

[54] B. Guo, G. Pan, Q. Guo et al., "Saccharides and temperature dual-responsive hydrogel layers for harvesting cell sheets," Chemical Communications, vol. 51, no. 4, pp. 644-647, 2015.

[55] J. Xu, D. Yang, W. Li, Y. Gao, H. Chen, and H. Li, "Phenylboronate-diol crosslinked polymer gels with reversible sol-gel transition," Polymer, vol. 52, no. 19, pp. 4268-4276, 2011.

[56] M. Piest, X. Zhang, J. Trinidad, and J. F. Engbersen, "pHresponsive, dynamically restructuring hydrogels formed by reversible crosslinking of PVA with phenylboronic acid functionalised PPO-PEO-PPO spacers (Jeffamines ${ }^{\circledR}$ )," Soft Matter, vol. 7, no. 23, pp. 11111-11118, 2011.

[57] M. C. Roberts, M. C. Hanson, A. P. Massey, E. A. Karren, and P. F. Kiser, "Dynamically restructuring hydrogel networks formed with reversible covalent crosslinks," Advanced Materials, vol. 19, no. 18, pp. 2503-2507, 2007. 